\title{
Recalculating the quasar luminosity function of the extended Baryon Oscillation Spectroscopic Survey
}

\author{
David M. Caditz
}

\author{
633 Main St, Sausalito, CA 94965, USA \\ e-mail: dcaditz@gmail.com
}

Received 28 August 2017 / Accepted 27 September 2017

\begin{abstract}
Aims. The extended Baryon Oscillation Spectroscopic Survey (eBOSS) of the Sloan Digital Sky Survey provides a uniform sample of over 13000 variability selected quasi-stellar objects (QSOs) in the redshift range $0.68<z<4.0$. The previously published QSO luminosity function (QLF), parametric model fits, and projected number counts based on this survey may be in error because the $k$-correction has apparently been misapplied, which results in underestimating the intrinsic brightness of roughly half of the eBOSS sources. This work provides new estimates of the QLF based on a corrected eBOSS dataset.

Methods. Intrinsic luminosities were recalculated using the appropriate $g$-band $k$-correction function. The QLF was determined for the corrected dataset using a model-weighted estimator, and parametric models were refit to the corrected luminosity function. Projected number counts based on the corrected models are also provided.

Results. At redshifts higher than the "pivot" redshift, $z_{\mathrm{p}}=2.2$, the original and recalculated results differ significantly; in particular, the new results show stronger high-redshift evolution in the best-fit models than the original eBOSS analysis. A new seven-parameter QLF model is provided that fits the corrected eBOSS dataset.
\end{abstract}

Key words. quasars: general - galaxies: active - galaxies: evolution - methods: data analysis - methods: statistical - surveys

\section{Introduction}

Palanque-Delabrouille et al. (2016; hereafter PD16) provide an estimate of the QSO luminosity function (QLF) based on data collected from the extended Baryon Oscillation Spectroscopic Survey (eBOSS) comprising 13876 spectroscopically confirmed QSOs with redshift $0.68<z<4.0$ (Dawson et al. 2016). The derived QLF has the well-known double power-law (DPL) shape with a break magnitude that evolves with redshift. This QLF is well fit by a pure luminosity evolution (PLE) model at least up to a so-called "pivot" redshift, $z_{\mathrm{p}}=2.2$. Above $z_{\mathrm{p}}$, the QLF may show some departure from PLE with possible variations in DPL slope and QLF normalization. Parametric models fit to the eBOSS dataset show an overall brightening of the QSO population up to $z_{\mathrm{p}}$ and then a constant brightness or dimming population up to $z=4$. This result suggests that some interesting phenomena occur at the pivot redshift $z_{\mathrm{p}}$, which may have important consequences for physical modeling of QSO formation and energy production as well as for determining expected number counts for future QSO surveys.

In this paper I report on an apparent error in the eBOSS data analysis that affects the shape and the evolution of the QLF, particularity at redshifts $z>z_{\mathrm{p}}$. The corrected dataset places tighter constraints on the high-redshift QLF and indicates a stronger luminosity evolution than found in the original analysis. These corrected results are important for researchers attempting to derive physical models for QSO energy production, projecting number counts for future surveys, and for modeling the contribution of QSOs to background radiation.

\section{2. eBOSS dataset}

The eBOSS QSO dataset comprising 13876 sources with redshift, $z$, apparent $g$-magnitude (corrected for galactic reddening), and absolute magnitude (normalized to $z=2$ ), $M_{g}$, was kindly provided by N. Palanque-Delabrouille. Absolute magnitude, apparent magnitude, and redshift are related by

$M_{g}=g-D_{M}(z)-K(M, z)$,

where $D_{M}(z)$ is the distance modulus. I assume a standard flat $\Lambda \mathrm{CDM}$ cosmology $\left(\Omega_{M}, \Omega_{\Lambda}, w, h\right)=(0.6935,0.3065,-1$, 0.679 ), consistent with PD16. The $k$-correction, $K(M, z)$, (normalized to $z=2$ ) accounts for redshifting of the observed bandpass and absorption due to intervening mass, and is dependent on the spectral energy distribution of the source. Since QSOs are known to have luminosity-dependent spectral properties, the $k$-correction function exhibits a degree of dependence on magnitude (McGreer et al. 2013).

The $g$-band $k$-correction function adopted by PD16 is shown in their Fig. 9. This $k$-correction function is attributed to McGreer et al. (2013), and is consistent with the $g$-band $k$-correction function provided by Richards et al. (2006). For comparison, an analytic $k$-correction function from Croom et al. (2009) is also shown. For redshifts $z \lesssim 3$, these functions differ by no more than approximately $0.2 \mathrm{mag}$. At higher redshifts, the functions differ by more than 1 mag because the effect of intervening Lyman- $\alpha$ absorbers has not been accounted for by Croom et al. (2009), who only considered sources up to redshift $z=2.6$.

The $k$-correction function actually used for the PD16 eBOSS dataset can be extracted from the data in a straightforward 


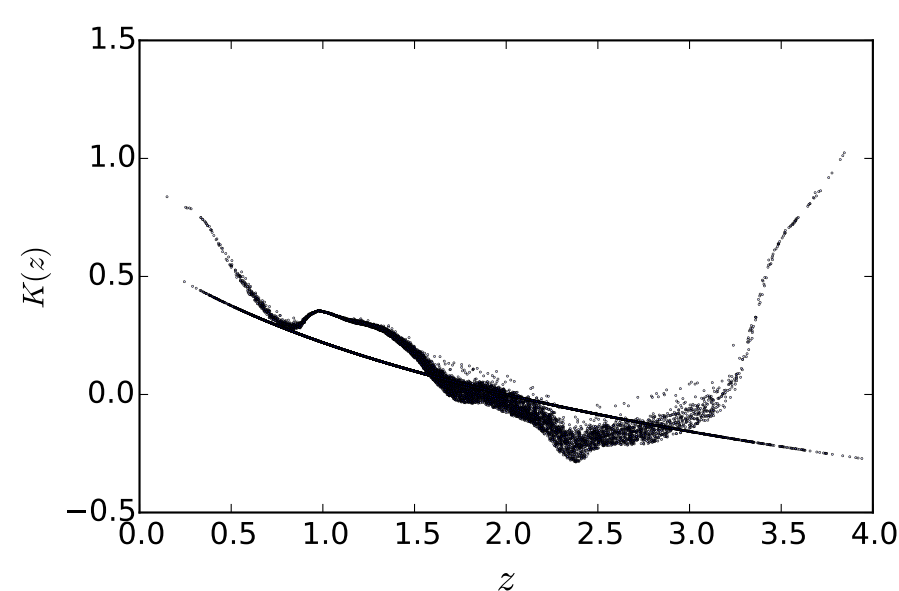

Fig. 1. $k$-correction extracted from the original eBOSS dataset that was used to calculate the QLF by Palanque-Delabrouille et al. (2016; compare with their Fig. 9). The incorrect $k$-correction function was apparently applied to roughly half of the sources.

manner using Eq. (1). The results of this extraction are provided in Fig. 1. This figure reveals that two different $k$-correction functions were applied to portions of the dataset. The Croom et al. (2009) $k$-correction was apparently mistakenly applied to a large number of sources. This result is in contradiction to the data reduction process described in PD16, which states that the McGreer et al. (2013) function was used, and indicates an apparent error that affects the resulting QLF calculations. The effect is greatest at high redshifts where the $k$-correction functions differ by significantly more than the magnitude bin width.

\section{QSO luminosity function}

I recalculated eBOSS absolute magnitudes applying a single $k$-correction function to all sources. Lacking access to the detailed $k$-correction used by PD16, I estimated the $k$-correction function by fitting a spline curve to the portion of the dataset to which the McGreer et al. (2013) function had been correctly applied. This eliminates the magnitude-dependence of the $k$ correction function and may introduce small errors in magnitude, but such errors are minor compared to those caused by applying the incorrect $k$-correction.

I then repeated the remaining data analysis procedures of PD16 to calculate the QLF based on the corrected magnitudes. The data were divided into identical redshift and magnitude bins as used by PD16: redshift bins with limits $z=(0.68,1.06$, $1.44,1.82,2.2,2.6 .3 .0,3.5,4.0)$ and absolute magnitude bins of width $\Delta M_{g}=0.4$. The QLF was recalculated using the modelweighted estimator described by Miyaji et al. (2001) that was previously used by Eales (1993) in the context of galaxy luminosity functions:

$\Phi\left(M_{g i}, z_{i}\right)=\Phi_{\bmod }\left(M_{g i}, z_{i}\right) \frac{N_{\mathrm{obs}, i}}{N_{\bmod , i}}$

The subscript $i$ refers to the bin centered on $\left(M_{g i}, z_{i}\right)$, and $\Phi_{\bmod }$ is a model QLF,

$N_{\text {mod }, i}=\iint_{\operatorname{bin} i} \Phi_{\bmod }\left(M_{g}, z\right) \mathrm{d} M_{g} \frac{\mathrm{d} V}{\mathrm{~d} z} \mathrm{~d} z$

is the number of sources expected in bin $i$ according to the model, and $N_{\mathrm{obs}, i}$ is the observed (completeness-corrected) number count for bin $i$. Since detailed information regarding the

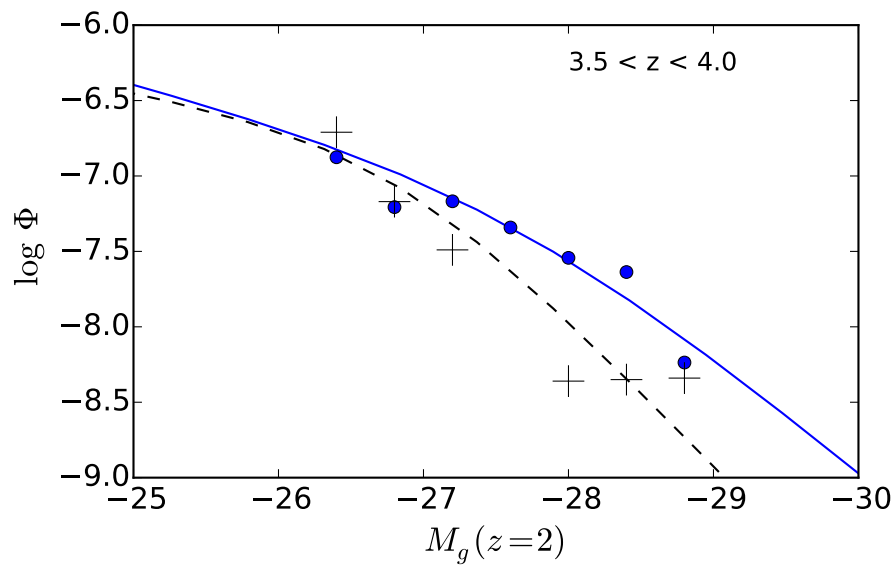

Fig. 2. Comparison of QLFs for the redshift bin $3.5<z<4.0$. Plus signs indicate the QLF reported by Palanque-Delabrouille et al. (2016). Filled circles show the QLF based on the corrected eBOSS dataset. The corrected QLF shows a shallower slope than the original version. The dashed curve is the best-fit PLE+LEDE model reported by Palanque-Delabrouille et al. (2016). The solid curve shows the best-fit PLE+LEDE model based on the corrected dataset.

eBOSS completeness is not available, an average completeness function was used (PD16, Fig. 8).

For the model QLF in Eq. (2), I used the PLE+LEDE model described by PD16. The initial model parameters values are taken as the optimal values provided in PD16, Table 4. The final solution was obtained through interative application of Eq. (2) and $\chi^{2}$ optimization of the model parameters until convergence was obtained.

Figure 2 compares the original and recalculated QLFs for the highest redshift bin $3.5<z<4.0$ where the different $k$-correction treatments are expected to have the greatest impact. Plus signs indicate the QLF reported by PD16. Filled circles show the recalculated QLF based on the corrected eBOSS data. The recalculated QLF shows a shallower slope than the original version and extends to one magnitude bin, or 0.4 magnitudes, dimmer. The dashed curve is the best-fit PLE+LEDE model QLF reported by PD16. The solid curve shows the best-fit PLE+LEDE model QLF based on the corrected dataset (model results are described in more detail below). The model DPL break magnitude is brighter for the corrected dataset, indicating a relatively stronger luminosity evolution at high redshifts than was reported by PD16. This result is consistent with DPL break evolution found by Ross et al. (2013) for the redshift range $z_{\mathrm{p}}<z<3.5$.

\section{Parametric fits}

The PLE-Pivot ${ }^{1}$ and PLE+LEDE models used to fit the eBOSS QLF are defined in PD16, Sect. 5.2. Both models have ten free parameters that describe the DPL slopes, and the normalization and evolution of the characteristic magnitude on either side of the fixed pivot redshift, $z_{\mathrm{p}}=2.2$. To these I added a third model defined as follows: the bright and dim power-law slopes are characterized by the parameters $\alpha$ and $\beta$, respectively. The pivot redshift, $z_{\mathrm{p}}$, is a free parameter. The QLF normalization is allowed 1 I use the term PLE-Pivot to account for the change in shape of the
QLF at $z_{\mathrm{p}}$. A true PLE model does not change shape. 


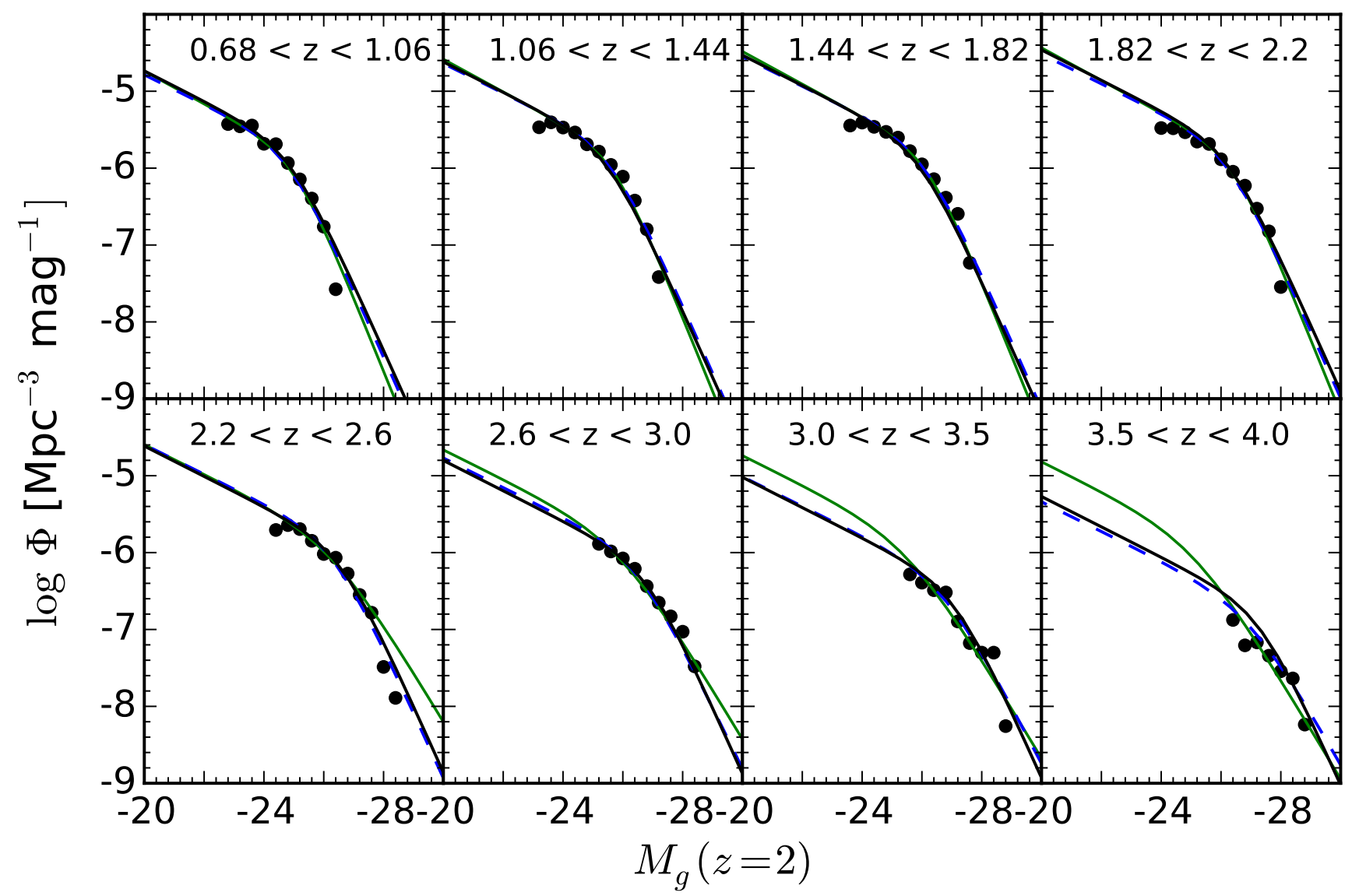

Fig. 3. Binned QLF (filled circles) and best-fit PLE+LEDE (dashed), PLE-Pivot (solid green), and PLE+LEDE2 (solid black) models based on the corrected eBOSS dataset.

to vary above $z_{\mathrm{p}}$ according to

$\log \left[\Phi^{*}(z)\right]= \begin{cases}\log \left[\Phi^{*}\right] & z<z_{\mathrm{p}} \\ \log \left[\Phi^{*}\right]+c_{1}\left(z-z_{\mathrm{p}}\right) & z>z_{\mathrm{p}}\end{cases}$

Evolution of the DPL break magnitude is given by the smooth and continuous function:

$M^{*}(z)=M^{*}-c_{2} 2.5 \log (1+z-\sqrt{1+z})$.

The model thus comprises seven parameters: $\left(\alpha, \beta, M^{*}, \Phi^{*}, c_{1}\right.$, $c_{2}$, and $\left.z_{\mathrm{p}}\right)$. Like the PLE+LEDE model, this model undergoes PLE below redshift $z_{\mathrm{p}}$ and both luminosity and density evolution at redshifts higher than $z_{\mathrm{p}}$. This model is termed PLE+LEDE2.

These models were fit to the corrected eBOSS dataset by minimizing the $\chi^{2}$ residuals between the model and observed (completeness corrected) number counts summed over bins. Bins with model counts $N_{\text {mod, } i}<1$ we excluded from the fit, as were a small number of bins near the survey flux limit with apparently anomalous counts. These anomalies are likely caused by application of average rather than detailed completeness corrections. Best-fit models are shown for each redshift bin in Fig. 3. Best-fit parameter values are given in Table 1. I also list in Table 1 the original parameters reported by PD16 for the PLEPivot and PLE+LEDE models. Inspection of Table 1 shows that the original and recalculated fits differ significantly for $z>z_{\mathrm{p}}$. In addition, there are fewer anomalous bins in the recalculated dataset, as can be seen from the $\chi^{2} / n$ values.

Predictions for the evolution of the DPL break magnitude, $M^{*}$, with redshift are plotted in Fig. 4 for the original (dashed) and corrected eBOSS PLE+LEDE models and the PLE+LEDE2 model (solid red and black, respectively), projected to $z=5$. I also show the results of Richards et al. (2005), Croom et al. (2009), and Ross et al. (2013). The corrected eBOSS models show a stronger evolution than the original, consistent with previous works (e.g., Ross et al. 2013). The discontinuity in slope for the PLE+LEDE model results from the parametric model, which enforces continuity at $z_{\mathrm{p}}$, but not smoothness. The PLE+LEDE2 model is smooth at all redshifts.

\section{Projected number counts}

Table 2 provides a recalculation of the projected QSO number counts for $0<z<6$ and $15.5<m_{g}<25$ for a hypothetical survey covering $10000 \mathrm{deg}^{2}$. The table shows counts for both the PLE-Pivot and PLE+LEDE models with parameters optimized to the corrected eBOSS dataset. The values shown are raw counts to which a survey completeness function has not been applied. The counts should be reduced by a factor determined by the appropriate completeness function in order to obtain the expected number counts for a given survey. The PLE-Pivot and PLE+LEDE counts are comparable up to $z \sim 3$, reflecting the similarity of the QLFs in this redshift range. At higher redshifts the PLE-Pivot model predicts significantly higher counts. This behavior is expected based on the QLFs shown in Fig. 3. For example, in the highest redshift bin, the dim end of the PLEPivot QLF is a factor of about three higher than the PLE+LEDE QLF, resulting in a similar factor in the number counts. At higher 
Table 1. Model parameters.

\begin{tabular}{|c|c|c|c|c|c|c|}
\hline Dataset & Redshift & \multicolumn{4}{|c|}{ Parameters } & $\chi^{2} / n$ \\
\hline \multicolumn{7}{|c|}{ PLE-Pivot } \\
\hline Original & $\begin{array}{l}0.68<z<4.0 \\
0.68<z<2.2 \\
2.2<z<4.0\end{array}$ & $\begin{array}{l}M_{g}^{*}\left(z_{\mathrm{p}}\right) \\
-26.71 \pm 0.15 \\
\alpha \\
-4.31 \pm 0.26 \\
-3.04 \pm 0.12\end{array}$ & $\begin{array}{l}\log \Phi^{*} \\
-6.01 \pm 0.07 \\
\beta \\
-1.54 \pm 0.04 \\
-1.38 \pm 0.07\end{array}$ & $\begin{array}{l}k_{1} \\
-0.08 \pm 0.08 \\
-0.25 \pm 0.09\end{array}$ & $\begin{array}{l}k_{2} \\
-0.40 \pm 0.05 \\
-0.05 \pm 0.06\end{array}$ & $135 / 76$ \\
\hline Corrected & $\begin{array}{l}0.68<z<4.0 \\
0.68<z<2.2 \\
2.2<z<4.0\end{array}$ & $\begin{array}{l}M_{g}^{*}\left(z_{\mathrm{p}}\right) \\
-26.50 \pm 0.04 \\
\alpha \\
-3.40 \pm 0.19 \\
-2.62 \pm 0.12\end{array}$ & $\begin{array}{l}\log \Phi^{*} \\
-5.81 \pm 0.01 \\
\beta \\
-1.53 \pm 0.02 \\
-1.48 \pm 0.05\end{array}$ & $\begin{array}{l}k_{1} \\
-0.03 \pm 0.02 \\
-0.36 \pm 0.04\end{array}$ & $\begin{array}{l}k_{2} \\
-0.35 \pm 0.02 \\
0.01 \pm 0.03\end{array}$ & $277 / 98$ \\
\hline \multicolumn{7}{|c|}{ PLE+LEDE } \\
\hline Original & $\begin{array}{l}0.68<z<4.0 \\
0.68<z<2.2 \\
2.2<z<4.0\end{array}$ & $\begin{array}{l}M_{g}^{*}(0) \\
-22.25 \pm 0.49 \\
k_{1} \\
1.59 \pm 0.28 \\
c_{1 a} \\
-0.46 \pm 0.10 \\
\end{array}$ & $\begin{array}{l}\log \Phi^{*}(0) \\
-5.93 \pm 0.07 \\
k_{2} \\
-0.36 \pm 0.09 \\
c_{1 b} \\
-0.06 \pm 0.10\end{array}$ & $\begin{array}{l}\alpha \\
-3.89 \pm 0.23\end{array}$ & $\begin{array}{l}\beta \\
-1.47 \pm 0.06\end{array}$ & $146 / 77$ \\
\hline Corrected & $\begin{array}{l}0.68<z<4.0 \\
0.68<z<2.2 \\
2.2<z<4.0\end{array}$ & $\begin{array}{l}M_{g}^{*}(0) \\
-22.01 \pm 0.04 \\
k_{1} \\
1.67 \pm .01 \\
c_{1 a} \\
-0.44 \pm 0.04\end{array}$ & $\begin{array}{l}\log \Phi^{*}(0) \\
-5.72 \pm 0.02 \\
k_{2} \\
-0.41 \pm 0.01 \\
c_{1 b} \\
-0.12 \pm 0.04\end{array}$ & $\begin{array}{l}\alpha \\
-3.18 \pm 0.15\end{array}$ & $\begin{array}{l}\beta \\
-1.48 \pm 0.02\end{array}$ & $242 / 100$ \\
\hline & & & PLE+LEDE2 & & & \\
\hline Corrected & & $\begin{array}{l}M_{g}^{*} \\
-25.95 \pm 0.04 \\
c_{1} \\
-0.59 \pm 0.03\end{array}$ & $\begin{array}{l}\log \Phi^{*} \\
-5.70 \pm 0.02 \\
c_{2} \\
1.37 \pm 0.08\end{array}$ & $\begin{array}{l}\alpha \\
-3.16 \pm 0.16 \\
z_{\mathrm{p}} \\
2.05 \pm 0.04\end{array}$ & $\begin{array}{l}\beta \\
-1.49 \pm 0.02\end{array}$ & $282 / 100$ \\
\hline
\end{tabular}

Notes. Original parameter values are those reported by Palanque-Delabrouille et al. (2016). Corrected values are optimized using the corrected eBOSS dataset. $90 \%$ confidence intervals for corrected dataset are determined by varying $\chi^{2}$ residuals for each parameter independently and do not account for parameter covariances. PLE-Pivot and PLE+LEDE model parameters are defined in Palanque-Delabrouille et al. (2016, Sect. 5.2).

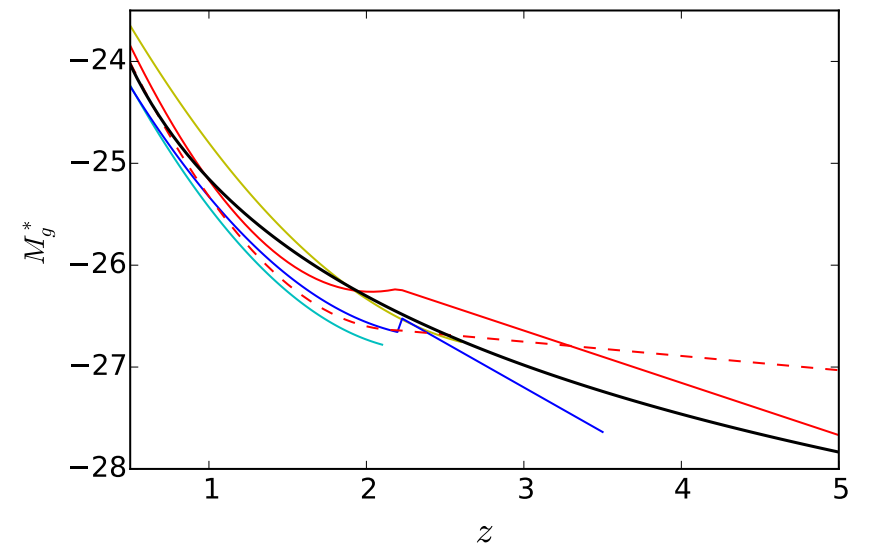

Fig. 4. Evolution of DPL break magnitude, $M_{g}^{*}(z)$, with redshift for various model luminosity functions. The solid red curve is the bestfit PLE+LEDE model from this paper. The dashed curve is the PLE+LEDE fit from Palanque-Delabrouille et al. (2016). The thicker black curve is the best-fit PLE+LEDE2 model from this paper. The cyan, yellow, and blue curves are from Richards et al. (2005), Croom et al. (2009), and Ross et al. (2013), respectively. redshifts, the difference increases, up to a factor of about ten at $z \sim 5$. This result is expected to hold qualitatively for different choices of $k$-correction and completeness functions.

\section{Conclusion}

The shape and evolution of the QLF determined from the eBOSS dataset can provide important information regarding QSO energy production, creation and destruction rates, fueling rates, and the local QSO environment. This work improves on the analysis of the eBOSS QLF by PD16 by correcting an apparent error that was due to improper application of the $k$-correction function. New QLF estimates based on a corrected eBOSS dataset are provided. The new estimates are consistent with previous estimates for redshifts $z \lesssim 2$ where the $k$-correction error is small. At higher redshifts, the corrected models are brighter than the original models. The corrected PLE+LEDE model shows stronger luminosity evolution and a shallower bright-end slope than the PD16 model. The new PLE-Pivot model shows a less drastic dimming with redshift above $z_{\mathrm{p}}$. The new results are consistent 
Table 2. Predicted raw number counts.

\begin{tabular}{|c|c|c|c|c|c|c|c|}
\hline \multicolumn{8}{|c|}{ PLE-Pivot } \\
\hline$g / z$ & 0.5 & 1.5 & 2.5 & 3.5 & 4.5 & 5.5 & Total \\
\hline 15.75 & 231 & 31 & 88 & 7 & 0 & 0 & 357 \\
\hline 16.25 & 491 & 94 & 193 & 15 & 0 & 0 & 793 \\
\hline 16.75 & 1041 & 282 & 414 & 32 & 0 & 0 & 1769 \\
\hline 17.25 & 2193 & 839 & 890 & 68 & 1 & 0 & 3991 \\
\hline 17.75 & 4509 & 2437 & 1912 & 142 & 2 & 0 & 9002 \\
\hline 18.25 & 9031 & 6756 & 4092 & 296 & 5 & 0 & 20180 \\
\hline 18.75 & 16839 & 17124 & 8607 & 614 & 10 & 1 & 43195 \\
\hline 19.25 & 28493 & 37655 & 17314 & 1255 & 22 & 2 & 84741 \\
\hline 19.75 & 43408 & 69653 & 32157 & 2516 & 47 & 6 & 147787 \\
\hline 20.25 & 60916 & 110247 & 54322 & 4907 & 100 & 12 & 230504 \\
\hline 20.75 & 81360 & 156914 & 83974 & 9206 & 209 & 27 & 331690 \\
\hline 21.25 & 105981 & 210645 & 121555 & 16450 & 436 & 57 & 455124 \\
\hline 21.75 & 136475 & 274914 & 167951 & 27811 & 902 & 120 & 608173 \\
\hline 22.25 & 174859 & 354284 & 224569 & 44422 & 1841 & 251 & 800226 \\
\hline 22.75 & 223554 & 454068 & 293542 & 67289 & 3683 & 523 & 1042659 \\
\hline 23.25 & 285547 & 580593 & 377942 & 97331 & 7155 & 1077 & 1349645 \\
\hline 23.75 & 364588 & 741633 & 481919 & 135474 & 13345 & 2185 & 1739144 \\
\hline 24.25 & 465430 & 946942 & 610828 & 182787 & 23630 & 4334 & 2233951 \\
\hline 24.75 & 594124 & 1208872 & 771430 & 240689 & 39403 & 8313 & 2862831 \\
\hline Total & 2599070 & 5173983 & 3253699 & 831311 & 90791 & 16908 & 11965762 \\
\hline \multicolumn{8}{|c|}{ PLE+LEDE } \\
\hline$g / z$ & 0.5 & 1.5 & 2.5 & 3.5 & 4.5 & 5.5 & Total \\
\hline 15.75 & 221 & 68 & 25 & 5 & 0 & 0 & 318 \\
\hline 16.25 & 469 & 178 & 64 & 11 & 1 & 0 & 722 \\
\hline 16.75 & 992 & 466 & 163 & 25 & 1 & 0 & 1648 \\
\hline 17.25 & 2076 & 1211 & 416 & 58 & 3 & 1 & 3764 \\
\hline 17.75 & 4233 & 3091 & 1054 & 134 & 6 & 1 & 8518 \\
\hline 18.25 & 8400 & 7628 & 2625 & 307 & 12 & 2 & 18974 \\
\hline 18.75 & 15655 & 17707 & 6328 & 691 & 23 & 4 & 40408 \\
\hline 19.25 & 26856 & 37253 & 14379 & 1511 & 47 & 6 & 80052 \\
\hline 19.75 & 41808 & 68835 & 29757 & 3142 & 94 & 11 & 143646 \\
\hline 20.25 & 59520 & 111061 & 54516 & 6083 & 184 & 20 & 231384 \\
\hline 20.75 & 79407 & 160307 & 87958 & 10767 & 351 & 34 & 338822 \\
\hline 21.25 & 101854 & 214838 & 127642 & 17342 & 640 & 55 & 462371 \\
\hline 21.75 & 127923 & 275960 & 172185 & 25682 & 1106 & 88 & 602943 \\
\hline 22.25 & 158977 & 346746 & 222351 & 35630 & 1795 & 135 & 765633 \\
\hline 22.75 & 196566 & 430992 & 280390 & 47213 & 2728 & 198 & 958086 \\
\hline 23.25 & 242458 & 532933 & 349275 & 60711 & 3906 & 279 & 1189561 \\
\hline 23.75 & 298723 & 657372 & 432431 & 76632 & 5326 & 381 & 1470865 \\
\hline 24.25 & 367851 & 809934 & 533776 & 95670 & 7008 & 506 & 1814745 \\
\hline 24.75 & 452862 & 997366 & 657905 & 118687 & 8996 & 657 & 2236474 \\
\hline Total & 2186851 & 4673945 & 2973242 & 500298 & 32227 & 2379 & 10368943 \\
\hline
\end{tabular}

Notes. Predicted raw QSO number counts for a survey covering $10000 \mathrm{deg}^{2}$ to redshift $z=6$ and magnitude $g<25$ for the PLE-Pivot and PLE+LEDE models based on models fit to the corrected eBOSS dataset. 
with previous studies, which found similar evolution and slopes at redshifts $z>z_{\mathrm{p}}$.

A kink in the evolution of $M^{*}(z)$ in the PLE+LEDE model suggests that a more satisfactory evolutionary model could be proposed. I have provided a new seven-parameter PLE+LEDE2 model with a smooth and continuous $M^{*}(z)$ parameterization that fits the corrected eBOSS data nearly as well as the ten-parameter PLE+LEDE model of PD16. The motivation for this new parameterization will be discussed in a future paper.

The evolution of the QLF for redshifts $z<4$ can be used to estimate number counts for future deep surveys. Table 2 provides projected number counts to $z=6$ (without reductions that are due to survey completeness effects). In contrast to the projected counts in PD16, the PLE-Pivot and PLE-LEDE models differ significantly for dim high-redshift bins. This is due to the relatively higher normalization of the PLE-Pivot model at high redshifts, as can be seen in Fig. 3. Because the DPL break magnitude is already observable for the PLE+LEDE model at $z \sim 4$, the PLE+LEDE results can be taken as minimum projected number counts, barring an unexpected change in QLF shape. Future surveys may place greater restrictions on the high-redshift behavior of the QLF and enable us to distinguish between PLEPivot, PLE+LEDE, PLE+LEDE2, and similar models.

Acknowledgements. I would like to thank Dr. N. Palanque-Delabrouille for providing access to the eBOSS data and for her consultation regarding this work.

\section{References}

Croom, S. M., Richards, G. T., Shanks, T., et al. 2009, MNRAS, 399, 1755 Dawson, K. S., Kneib, J.-P., Percival, W. J., et al. 2016, AJ, 151, 44 Eales, S. 1993, ApJ, 404, 51

McGreer, I. D., Jiang, L., Fan, X., et al. 2013, ApJ, 768, 105

Miyaji, T., Hasinger, G., \& Schmidt, M. 2001, A\&A, 369, 49

Palanque-Delabrouille, N., Magneville, C., Yèche, C., et al. 2016, A\&A, 587, A41

Richards, G. T., Croom, S. M., Anderson, S. F., et al. 2005, MNRAS, 360, 839

Richards, G. T., Strauss, M. A., Fan, X., et al. 2006, ApJ, 131, 2766

Ross, N. P., McGreer, I. D., White, M., et al. 2013, ApJ, 773, 14 